\title{
Paralisia do nervo oculomotor como manifestação inicial de hematoma subdural crônico - Relato de caso
}

\author{
Carlos Umberto Pereira ${ }^{1}$, José Anísio Santos Júnior², Ana Cristina Lima Santos²
}

Universidade Federal de Sergipe e Hospital de Urgências de Sergipe, Aracaju, SE, Brasil.

\section{RESUMO}

A presença de paralisia do nervo oculomotor (NOM) sem outro déficit neurológico é considerada rara como forma de apresentação em hematoma subdural crônico (HSDC). Geralmente apresenta sintomas de déficit neurológico focal, cefaleia e alterações do nível de consciência, havendo múltiplos diagnósticos diferenciais. RTA, 79 anos, masculino. Paciente com demência senil, hipertensão arterial sistêmica e diabetes mellitus. Estado geral: moderado. Exame físico: sonolento, eupneico. Exame neurológico: disfásico e anisocoria esquerda. Tomografia computadorizada (TC) de crânio sem contraste revelou lesão com densidade heterogênea na região frontoparietotemporal esquerda com efeito de massa e hipodensa na região frontoparietal direita. Submetido a trépano-punção frontal anterior e parietal posterior esquerda e drenagem do hematoma. Evoluiu com melhora da paralisia do NOM à esquerda. Em caso de HSDC volumoso, pode-se comprimir o mesencéfalo e apresentar-se herniação do uncus do hipocampo, podendo causar paralisia do NOM. Seu diagnóstico precoce e tratamento correto apresentam bons resultados.

\section{PALAVRAS-CHAVE}

Hematoma subdural crônico, doenças do nervo oculomotor, terapêutica.

\section{ABSTRACT}

Oculomotor nerve palsy as initial manifestation of chronic subdural hematoma - Case report The presence of complete paralysis of the oculomotor nerve (OMN) with no other neurological deficit is rare as the presentation of chronic subdural hematoma (CSDH). Usually there are symptoms of focal neurologic deficit, headache and changing consciousness level, so there are multiple differential diagnoses. RTA, 79-year-old man. Patient who has senile dementia, hypertension and diabetes mellitus. General condition: moderate. Physical examination: sleepy, eupneic. Neurological examination: dysphasia and anisocoria left eye. Computed tomography (CT) scan without contrast revealed a lesion with heterogeneous density in the left frontoparietotemporal region, with mass effect and hipodense region right parietofrontal. The patient was submited to trepano-punction at left anterior frontal and posterior parietal and drainage of the hematoma. He evolved with neurological improvement of the paralysis of $\mathrm{OMN}$ on the left eye. The CSDH when it was large, can compress the midbrain and provide herniation of hippocampus' uncus may cause paralysis of the OMN. Early diagnosis and correct treatment has shown good results.

\section{KEYWORDS}

Hematoma subdural chronic, oculomotor nerve diseases, therapeutics.

1 Professor adjunto doutor do Departamento de Medicina da Universidade Federal de Sergipe (UFS), neurocirurgião do Serviço de Neurocirurgia do Hospital de Urgência de Sergipe (HUSE), Aracaju, SE, Brasil.

2 Acadêmicos de Medicina da UFS, Aracaju, SE, Brasil. 


\section{Introdução}

O hematoma subdural crônico (HSDC) é uma das entidades mais frequentes da neurocirurgia, e os seus diagnósticos diferenciais incluem demência, meningite e abscessos. ${ }^{1}$ Classicamente, o HSDC é definido como uma coleção sanguínea, com grau variado de degeneração, encapsulado, de evolução crônica, localizado entre a dura-máter e a aracnoide. ${ }^{2-5}$

A presença de paralisia do nervo oculomotor (NOM) sem outro déficit neurológico é considerada rara como forma de apresentação em HSDC, ${ }^{6,7}$ sendo as manifestações clínicas do HSDC múltiplas e complexas, podendo simular outros processos patológicos. ${ }^{5,8,9}$ Pode-se manifestar com crises epilépticas ${ }^{10,11} \mathrm{e}$ variados graus de confusão mental ou coma. ${ }^{11}$ Alguns pacientes são considerados como portadores de distúrbios psiquiátricos por causa dos sintomas depressivos e paranoides. ${ }^{2}$

Os autores apresentam um caso de HSDC cuja manifestação clínica inicial foi de paralisia do NOM. Eles discutem sua fisiopatologia e conduta.

\section{Relato do caso}

RTA, 79 anos, masculino. Paciente com história de demência senil, portador de hipertensão arterial sistêmica e diabetes mellitus. Estado geral: moderado. Exame físico: péssimo estado geral, eupneico. Exame neurológico: sonolento, anisocoria esquerda. Tomagrafia computadorizada (TC) de crânio sem contraste: lesão com densidade heterogênea na região frontoparietotemporal bilateral com maior coleção subdural à esquerda (Figuras 1 e 2), compatível tomograficamente

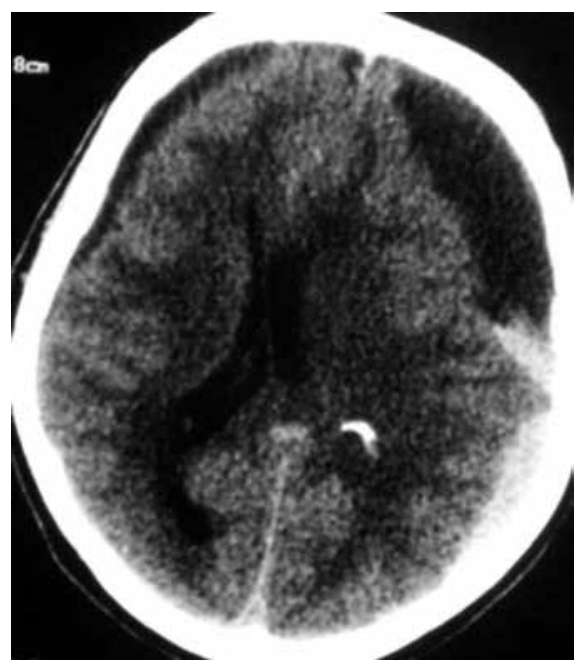

Figura 1 - TC de crânio sem contraste, demonstrando coleção subdural bilateral, desvio das estruturas da linha média. com hematoma subdural crônico. Submetido à trepanação frontal anterior e parietal posterior esquerda, à drenagem do hematoma e à lavagem da cavidade com soro fisiológico. Houve melhora do quadro neurológico e da paralisia do NOM à esquerda.

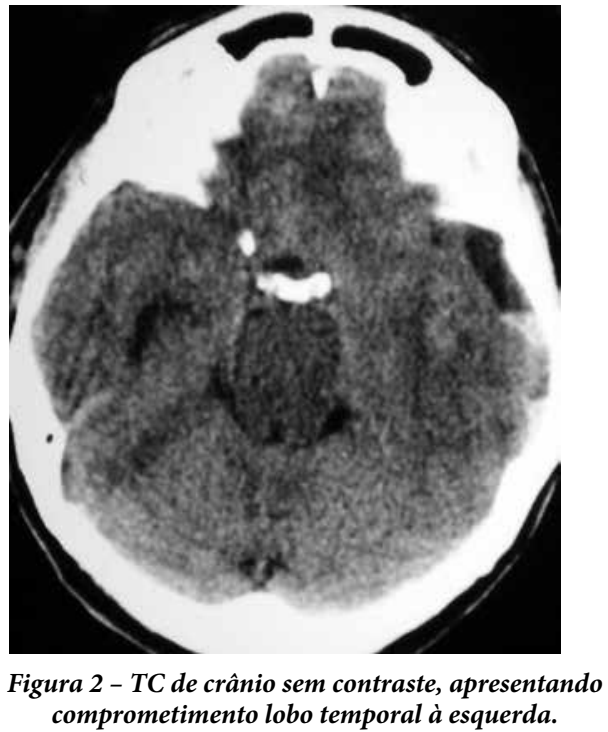

\section{Discussão}

O HSDC apresentando paralisia do NOM é incomum, sendo uma apresentação mais frequente em aneurismas intracranianos, diabetes mellitus e lesão no seio cavernoso. ${ }^{7}$ A lesão expansiva pelo HSDC pode provocar compressão do tronco cerebral por meio da herniação do uncus do lobo temporal, manifestando-se com midríase unilateral e déficit motor contralateral. ${ }^{12}$ O edema cerebral relacionado com herniação uncal exerce, ao longo da borda livre do tentório, pressão sobre o NOM, que pode levá-lo a uma perda de função. ${ }^{7} \mathrm{~A}$ herniação do uncus desvia a ponte para baixo, resultando no estiramento do NOM e no deslocamento das artérias cerebrais posteriores em um ângulo mais agudo; esse deslocamento comprime o NOM entre as artérias cerebral posterior e a cerebelar superior, provocando midríase e iridoplegia. ${ }^{1,4,13-16}$

O HSDC apresenta déficit neurológico focal em $58 \%$ dos casos, sendo a hemiparesia contralateral ao hematoma mais frequente, podendo ocorrer quadros de hemiplegias súbitas. ${ }^{2,3} \mathrm{~A}$ hemiparesia geralmente é por compressão do pedúnculo cerebral contra a borda livre do tentório exercida pelo hematoma. ${ }^{2}$ A cefaleia noturna e lateralizada varia entre $14 \%$ e $80 \%$ dos casos, sendo mais comum em adultos jovens do que em pacientes idosos. $^{2}$

As fibras que inervam os músculos do esfíncter pupilar estão localizadas na periferia e são, portanto, 
afetadas no início da compressão extrínseca do NOM como, por exemplo, nos casos de aneurisma da artéria comunicante posterior, tumores e hérnias cerebrais internas em casos de lesão expansiva. ${ }^{17-19}$

O exame de TC é útil no diagnóstico e conduta. ${ }^{20}$ Após a confirmação do diagnóstico, o tratamento pode ser conservador ou cirúrgico, sendo este último o mais indicado. ${ }^{2-4,21,22} \mathrm{O}$ tratamento conservador tem sido relatado como efetivo em pacientes portadores de HSDC pequenos e sem desvio de linha média. ${ }^{5,23,24}$ Em nosso paciente foi realizada trépano-punção unilateral com resolução do hematoma e melhora da paralisia do NOM.

As manifestações neurológicas do HSDC são várias, dependendo da evolução, volume e localização. O diagnóstico precoce e seu tratamento cirúrgico têm apresentado bom prognóstico.

\section{Referências}

1. Chen CC, Pai YM, Wang RF, Wang TL, Chong CF. Isolated oculomotor nerve palsy from minor head trauma. $\mathrm{Br} \mathrm{J}$ Sports Med. 2005;39(8):e34.

2. Adhiyaman V, Asghar M, Ganeshram KN, Bhowmick BK. Chronic subdural haematoma in the elderly. Postgrad Med J. 2002;78(916):71-5.

3. Barrios RS, Salazar LRM. Patobiología del hematoma subdural crónico. Electr J Biomed. 2008;2:65-71.

4. Hostalot-Panisello C, Carrasco-González A, BilbaoBarandica G, Pomposo-Gaztelu I, Garibi-Undabarrena JM. Chronic subdural haematoma. Presentation and therapeutic attitudes. Rev Neurol. 2002;35(2):123-7.

5. Reinges MH, Hasselberg I, Rohde V, Küker W, Gilsbach JM. Prospective analysis of bedside percutaneous subdural tapping for the treatment of chronic subdural haematoma in adults. J Neurol Neurosurg Psychiatry. 2000;69(1):40-7.

6. Ortega-Martínez M, Fernández-Portales I, Cabezudo JM, Rodríguez-Sánchez JA, Gómez-Perals LF, Giménez-Pando $J$. Isolated oculomotor palsy. An inusual presentation of chronic subural hematoma. Neurocirugia (Astur). 2003;14(5):423-5.

7. Phookan G, Cameron M. Bilateral chronic subdural haematoma: an unusual presentation with isolated oculomotor nerve palsy. J Neurol Neurosurg Psychiatry. 1994;57(9):1146.

8. Friede RL, Schachenmayr $\mathrm{W}$. The origin of subdural neomembranes. II. Fine structural of neomembranes. Am J Pathol. 1978;92(1):69-84.

9. Pereira CU. Hematoma subdural crônico no idoso. In: Pereira CU, Andrade AS, editores. Neurogeriatria. Rio de Janeiro: Revinter; 2000. p. 346-9.
10. Martínez F, Villar A. Hematoma subdural crónico: estudio de 58 pacientes consecutivos tratados en un mismo centro hospitalario. Rev Chil Neurocirug. 2008;31:14-23.

11. Martínez F. Presentación clínica del hematoma subdural crónico en adultos: el gran simulador. Rev Med Urug. 2007;23:92-8.

12. Rucker CW, Keefe WP, Kernohan JW. Pathogenesis of paralysis of the third cranial nerve. Trans Am Ophthalmol Soc. 1959;57:87-98.

13. Bhatti MT, Eisenschenk S, Roper SN, Guy JR. Superior divisional third cranial nerve paresis: clinical and anatomical observations of 2 unique cases. Arch Neurol. 2006;63(5):771-6.

14. Nakaguchi H, Tanishima T, Yoshimasu N. Factors in the natural history of chronic subdural hematomas that influence their postoperative recurrence. J Neurosurg. 2001;95(2):256-62.

15. Okuchi K, Fujioka M, Maeda Y, Kagoshima T, Sakaki T. Bilateral chronic subdural hematomas resulting in unilateral oculomotor nerve paresis and brain stem symptoms after operation - case report. Neurol Med Chir (Tokyo). 1999;39(5):367-71.

16. Milanes-Rodriguez G, Ibañez-Valdés L, Foyaca-Sibat $H$. Oculomotor nerve: clinical anatomy. Internet J Neurol. 2008;10(1): DOI: 10.5580/117.

17. Prasad S, Volpe NJ. Paralytic strabismus: third, fourth, and sixth nerve palsy. Neurol Clin. 2010;28(3):803-33.

18. Rabiu TB. Neuroclinical anatomy of the third cranial nerve. Internet J Neurol. 2010;12(2). DOI: 10.5580/242a.

19. Lee AG, Brazis PW. Third nerve palsies. In: Lee AG, Brazis $P W$, editors. Clinical pathways in neuro-ophthalmology: an evidence-based approach. $2^{\text {nd }}$ ed. New York: Thieme Medical Publishers; 2003. p. 253-80.

20. Senturk S, Guzel A, Bilici A, Takmaz I, Guzel E, Aluclu MU, et al. CT and MR imaging of chronic subdural hematomas: a comparative study. Swiss Med Wkly. 2010;140(2324):335-40.

21. Gallardo AJL, Esquivel JCE, Borroto RP. Hematoma subdural crónico. Resultados quirúrgicos en 2 años de trabajo. Rev Cubana Cir. 1999;38:57-61.

22. Kim JH, Kang DS, Kim JH, Kong MH, Song KY. Chronic subdural hematoma treated by small or large craniotomy with membranectomy as the initial treatment. J Korean Neurosurg Soc. 2011;50(2):103-8.

23. Göksu E, Akyüz M, Uçar T, Kazan S. Spontaneous resolution of a large chronic subdural hematoma: a case report and review of the literature. Ulus Travma Acil Cerrahi Derg. 2009;15(1):95-8.

24. Imai K. Rapid spontaneous resolution of signs of intracranial herniation due to subdural hematoma - case report. Neurol Med Chir (Tokyo). 2003;43(3):125-9.

Endereço para correspondência

Carlos Umberto Pereira

Av. Augusto Maynard, 245/404, Bairro São José

49015-380 - Aracaju, SE, Brasil

E-mail:umberto@infonet.com.br 